\title{
3 Research Square

\section{Elevated expression of FGB predicts poor survival in lung adenocarcinoma patients with tobacco exposure}

\section{Xu-gang Hu}

The 903rd hospital of PLA

\section{De-min Jiao}

The 903rd hospital of PLA

\section{You-liang Si}

The 903rd hospital of PLA

Jun Chen

The 903rd hospital of PLA

\section{Xia-li Tang}

The 903rd hospital of PLA

\section{Da-wei Wang}

The 903rd hospital of PLA

Qing-yong Chen ( $\nabla$ cqyong903@163.com )

\section{Research article}

Keywords: FGB; Prognosis; Lung adenocarcinoma; Tobacco exposure

Posted Date: October 17th, 2019

DOI: https://doi.org/10.21203/rs.2.16162/v1

License: (c) (1) This work is licensed under a Creative Commons Attribution 4.0 International License. Read Full License 


\section{Abstract}

Background: Tobacco exposure is the most important risk factor for the occurrence and death of lung cancer. Fibrinogen beta chain (FGB), the most abundant coagulation factor in plasma, is putatively involved in tumor progression. This study aimed to evaluate the expression pattern and prognostic value of FGB in lung adenocarcinoma with tobacco exposure. Methods: FGB expression in lung adenocarcinoma (LUAD) and corresponding normal lung tissues was compared in The Cancer Genome Atlas (TCGA) database. Stratified analysis was conducted to investigate the expression of FGB in LUAD patients with different tobacco exposure. Kaplan-Meier analysis and Cox-regression analysis were performed to evaluate the prognostic significance of FGB. The same survival analyses were conducted in the KM plotter database to validate the prognostic value of FGB. Results: FGB expression was significantly increased in LUAD tissues than in normal tissues $(P<0.001)$. The FGB expression in smoker group was higher than those in non-smoker group $(P<0.001)$. High FGB expression was associated with advanced $N$ stage $(P=0.033)$ and TNM stage $(P=0.046)$. The smoker group possessed higher level of FGB both in LUAD patients without $(P=0.003)$ or $(P=0.045)$ with lymph node metastasis. However, in early TNM stages, the smoker group showed elevated expression of FGB compared with non-smoker group $(P=0.0004)$, and in advanced TNM stages, there was no significant difference between the two groups $(P=0.350)$. In survival analyses, patients with high $F G B$ expression had remarkably worse overall survival $(P=0.007)$ and progression-free survival $((P=0.007)$ than those with low FGB expression. In smoker group, high level of FGB expression predicted worse OS $(P=0.003)$ and PFS $(P=0.029)$. Cox-regression analysis indicated that FGB was an independent prognostic factor for LUAD patients' OS ( $H R=1.616, P=0.017)$ and $P F S(H R=1.710, P=0.028)$. In smoking group, $F G B$ also served as a promising prognostic biomarker for OS $(H R=1.806, P=0.027)$ and $P F S(H R=2.181, P=0.013)$. Similar survival curves were generated in the KM plotter cohort. Conclusions: Our study demonstrated that elevated expression of FGB may identify an aggressive subgroup in LUAD with tobacco exposure and serve as an independent prognostic indicator in these patients.

\section{Background}

Lung cancer is the second most common (accounting for 12.9\%) type of cancer and the leading cause (accounting for 23.5\%) of cancer-related deaths worldwide.[1] In China, lung cancer is the most incident cancer and leading causes of cancer death, with 733,300 newly diagnosed cases and 610,200 deaths every year.[2] Lung adenocarcinoma (LUAD), with an increasing incident, is the major type of lung cancer, which accounts for nearly $40 \%$. Though the screening technology and therapy strategy has been made great advances, the early diagnosis rate and 5-year survival rate remains low.

Among the carcinogenic factors, tobacco exposure is confirmed to be the main cause of lung cancer. Epidemiological findings suggest that tobacco exposure was found in $90 \%$ of lung cancer patients, and contributed to approximately $16 \%$ of cancer diagnoses and $30 \%$ of cancer deaths.[3] The risk of lung cancer in tobacco exposed population is nearly 30 times higher than that in non-exposed population.[4] Due to the smoking cessation program, the lung cancer death rate has dropped steadily by approximately 
in $1.5 \%$ per year in United States.[1] However, smoking is still popular in China, especially in rural areas, and many people are exposed to secondhand or thirdhand smoke. Tobacco exposure causes the accumulation of toxic substances, such as benzo(a)pyrene and nicotine, induce DNA damage and genetic mutation in pulmonary epithelial cells, release of inflammatory mediators, reduction of cytochrome oxidase activity, suppression of immune system.[5-8]

Fibrinogen is a dimeric protein formed by three pairs of polypeptide chains ( $\mathrm{\otimes} \beta$ and $\gamma$ ), and is the most abundant coagulation factor in plasma. Fibrinogen beta chain (FGB) limits the assembly of fibrinogen. Elevated expression of FGB was associated with thrombus event such as stroke and acute coronary syndrome.[9-11] In recent years, more evidences showed that FGB was associated with tumor progression and poor prognosis in varies types of cancers. Suppressed expression of FGB by SIRT1 could inhibit RCC tumorigenesis. Higher levels of FGB were positively correlated to later TNM stages and higher tumor biomarkers in NSCLC.[12] Shu-Qi Li et al. reported that albumin-to-fibrinogen ratio could act as a prognostic biomarker of NSCLC patients, while the lower ratio predicted worse clinical outcome.[13]

In the present study, we aimed to identify promising biomarkers for LUAD with tobacco exposure via bioinformatics data mining. Our results showed that FGB was overexpressed in LUAD, especially those with tobacco exposure. Elevated expression of FGB was associated with tumor progression and worse overall survival and progression-free survival.

\section{Methods}

\section{TCGA lung adenocarcinoma cohort}

The lung adenocarcinoma cohort was downloaded from The Cancer Genome Atlas (TCGA) database (http://tcga-data.nci.nih.gov/tcga)[14]. In total, 439 cases of LUAD patients and 59 cases of normal with complete information were enrolled. The median age of the LUAD patients was 66 years. Among the subjects, 68 cases were never-smoker, 111 cases were current reformed smoker more than fifteen years, 153 cases were current reformed smoker less than fifteen years and 107 cases were current smoker. The non-smoker included that never smoker and current reformed smoker more than 15 years. The smoker included that current reformed smoker less than 15 years and current smoker.

\section{KM plotter}

The Kaplan-Meier Plotter database (http://kmplot.com/analysis) was queried to validate the prognostic significance of FGB mRNA expression[15]. The desired Affymetrix IDs is valid: 216238_s_at (FGB). The survival analysis was conducted in 720 cases of LUAD. The overall survival time and progression-free survival time were also calculated in LUAD patients stratified by smoking status. 


\section{Statistical analysis}

Statistical analysis was performed by using the SPSS statistical software package (standard version 22.0) and GraphPad Prism (version 8.0). All group data are presented as mean \pm SD. Two-group independent sample comparisons were preformed using Mann-Whitney $\mathrm{U}$ test for the KolmogorovSmirnov test showed the distribution of FGB expression was abnormal. Pearson chi-square test was applied to determine the relationship between FGB expression and the clinicopathological features. For survival analysis, Kaplan-Meier curve was generated with log-rank test. Multivariable Cox-regression analysis was applied to test for the independence of the prognostic value of FGB expression. $P<0.05$ was considered statistically significant.

\section{Results}

\section{Expression pattern of FGB in LUAD}

Comparing with the normal tissues, the LUAD tissues showed higher expression level of FGB mRNA $(P<0.001)$ (Fig. 1A). To further investigate the expression pattern of FGB in LUAD with tobacco exposure, stratified analysis was conducted in different exposure levels. The expression level of FGB in those reformed smoker more than fifteen years were similar to never smoker, and the FGB level in those reformed smoker less than fifteen years were similar to current smoker. However, the FGB expression in smoker group was much higher than those in non-smoker group $(\mathrm{P}<0.001)$ (Fig. 1B). These results show that the FGB expression level was significantly higher in LUAD, especially in those with tobacco exposure.

\section{Correlation between FGB expression and clinicopathological features}

The relationship between FGB expression and clinicopathological features was evaluated (Table 1). Besides the positive correlation between tobacco exposure, high level of FGB expression associated with advanced $N$ stage $(P=0.033)$ and TNM stage $(P=0.046)$ in LUAD. Stratified analysis revealed that both in LUAD patients without or with lymph node metastasis, the smoker group possessed higher level of FGB $(P=0.003$ and $P=0.045$, respectively) (Fig. $2 A)$. However, in early TNM stages, the smoker group showed elevated expression of FGB compared with non-smoker group $(P=0.0004)$, and in advanced TNM stages, there was no significant difference between the two groups $(P=0.350)$ (Fig. 2B). These results indicated that a positive correlation exists between FGB expression and LUAD progression.

\section{FGB serves as a prognostic biomarker in LUAD with tobacco exposure}

Kaplan-Meier analysis and Cox regression analysis were applied to evaluate the prognostic value of FGB in LUAD. Patients with high FGB expression had remarkably worse overall survival and progression-free survival than those with low FGB expression ( $P=0.007$ and $P=0.034$, respectively) (Fig. 3). Accordingly, the 
median OS time in $\mathrm{FGB}^{\text {high }}$ group (1081 (95\% Cl 733 to 1428) days) was shorter than in the $\mathrm{FGB}^{\text {low }}$ group ( 1622 (95\% Cl 1189 to 2054 ) days). Constantly, the median PFS time FGB ${ }^{\text {high }}$ group (680 (95\% Cl 407 to 952) days) was shorter than in the FGB ${ }^{\text {low }}$ group (1083 (95\% Cl 496 to 1669) days). Furtherly, stratified survival analysis was applied in LUAD patients with or without tobacco exposure. In the non-smoker group, patients' overall survival and progression-free survival were irrelevant to the expression of FGB (Fig. 4A and 4B). Nevertheless, in the smoker group, high level of FGB expression predicted worse OS $(\mathrm{HR}=1.727$ (95\% Cl 1.043 to 2.859), $\mathrm{P}=0.003)$ (Fig. 4C) and PFS ( $\mathrm{HR}=3.262(95 \% \mathrm{Cl} 1.805$ to 5.893$)$, $\mathrm{P}=0.029$ ) (Fig. 4D). Multivariable Cox-regression analysis showed that $\mathrm{FGB}$ was an independent prognostic factor for LUAD patients' OS (HR=1.616 (95\% $\mathrm{Cl} 1.090$ to 2.395), $\mathrm{P}=0.017)$ and PFS (HR= 1.710 (95\% Cl 1.060 to 2.759 ), $P=0.028$ ) (Table 2). In those LUAD patients with tobacco exposure, FGB also served as a promising prognostic biomarker for OS ( $\mathrm{HR}=1.806(95 \% \mathrm{Cl} 1.071$ to 3.044$), \mathrm{P}=0.027)$ and PFS (HR= 2.181 (95\% $\mathrm{Cl} 1.182$ to 4.023), $\mathrm{P}=0.013$ ) (Table 3).

To confirm the prognostic value of FGB in LUAD, the KM plotter online database was queried. Consistent with the results in TCGA cohort, high FGB expression was found to be correlated with worse OS (HR= 1.48 (95\% $\mathrm{Cl} 1.17$ to 1.87$), \mathrm{P}=0.00093)$ and $\mathrm{PFS}(\mathrm{HR}=1.78(95 \% \mathrm{Cl} 1.29$ to 2.44$), \mathrm{P}=0.00032)$ in LUAD patients (Fig. 5). Moreover, survival curves stratified by smoking status showed that upregulation of FGB was significantly associated with worse $O S(H R=1.73(95 \% \mathrm{Cl} 1.08$ to 2.78$), \mathrm{P}=0.021)$ and $\mathrm{PFS}(\mathrm{HR}=2.05$ ( $95 \% \mathrm{Cl} 1.31$ to 3.21$), \mathrm{P}=0.0013$ ) in LUAD patients with tobacco exposure, but not correlated with outcome in non-smokers (Fig. 6). These results suggest that FGB is a reproducible prognostic marker in LUAD patients, especially in those with tobacco exposure.

\section{Discussion}

Tobacco exposure is considered to be the main cause of cancer, chronic bronchitis, emphysema and cardiovascular diseases and claims the lives of more than 6 million people each year.[16] Alexandrov et al. examined mutational signatures and DNA methylation changes in 5243 cancers from 17 different types linked to smoking, and found the number of base substitutions was higher in smokers compared with nonsmokers, especially in lung epithelial cells.[17] High somatic mutations increased the risk of cancer incidence. A meta-analysis of human epidemiologic data conducted to evaluate the profile of common or therapy-targetable mutations in lung cancers demonstrated the smokers exhibited increased odds of KRAS mutation and decreased odds of EGFR and ALK-EML4 mutations, indicating that tobacco exposure limited survival benefits from target therapy.[18] Thus, an insight view of the effect and mechanism of tobacco exposure in lung cancer is urgently-needed.

Fibrinogen is a coagulation factor involved in coagulation, wound healing, inflammation, angiogenesis and cell adhesion. Recent studies have suggested that aberrant expression of FGB plays an important role in tumor progression.[19, 20] Palumo et al. reported that elevated expression of FGB is associated with metastasis and cancerous thrombosis. [5] In NSCLC patients, FGB expression predicts cancer associated thromboembolism and worse outcome.[21] 
In this study, we investigated the expression pattern and prognostic value of FGB in LUAD patients stratified by smoking status. We found that FGB expression was significantly increased in LUAD tissues, especially in those with tobacco exposure, indicating that smoking could stimulate the FGB pathway to change the biological characteristics of cancer cells. Interestingly, higher expression was found in the smoker group in early TNM stage patients, but not in advanced TNM stage patients. This result might due to statistical bias for a relatively small sample number of stage IV patients was involved in the study. The survival analyses showed that high expression of FGB was an independent prognostic indicator in LUAD patients with tobacco exposure, but not in those non-smokers, indicating that FGB might be developed as a therapeutic target for those patients.

The following issues would focus on: 1) the underlying molecular mechanism of FGB expression in LUAD patients with tobacco exposure. 2) the expression pattern of FGB in lung squamous cell carcinoma and small-cell carcinoma with tobacco exposure, for the two types of lung cancer are more relative to smoking.

\section{Conclusions}

We demonstrate that FGB expression levels are significantly elevated in LUAD patients with tobacco exposure. High expression of FGB correlates with tumor progression, and is an independent prognostic indicator in patients. Our results suggest that FGB could be used as a novel prognostic indicator and a potential therapeutic target in LUAD patients with tobacco exposure.

\section{Abbreviations}

FGB: Fibrinogen beta; TCGA: The cancer genome atlas; LUAD: Lung adenocarcinoma; OS: Overall survival; PFS: Progression-free survival; HR: Hazard ratio; Cl: confidence interval.

\section{Declarations}

\section{Ethics approval and consent to participate}

No ethics approval was required for this work. All data in this study are publicly available.

\section{Consent for publication}

Not applicable

\section{Availability of data and materials}


All data used in this study can be downloaded from TCGA data repository (http://tcgadata.nci.nih.gov/tcga) and Kaplan-Meier Plotter data repository (http://kmplot.com/analysis).

\section{Competing interests}

The authors declare that they have no competing interests.

\section{Funding}

The study was supported by Medical Health Science and Technology Project of Zhejiang Provincial Health Commission (No. 2019326546). The funding bodies were not involved in the design of this study, in the collection, analysis, and interpretation of the data, or in writing of the manuscript.

\section{Authors' contributions}

HXG, JDM and SYL contributed equally to this work. CQY served as guarantor of the paper and takes responsibility for the integrity of the work. HXG, JDM and TXL collected the data from TCGA. HXG, SLY and WDW performed the analysis. HXG and $\mathrm{CJ}$ drafted and revised the manuscript. All authors read and approved the final manuscript.

\section{Acknowledgements}

We would like to thank the peer reviewers for their constructive and insightful comments.

\section{References}

1. Siegel RL, Miller KD, Jemal A: Cancer statistics, 2019. CA Cancer J Clin 2019, 69(1):7-34.

2. Chen W, Zheng R, Baade PD, Zhang S, Zeng H, Bray F, Jemal A, Yu XQ, He J: Cancer statistics in China, 2015. CA Cancer J Clin 2016, 66(2):115-132.

3. Jacobs EJ, Newton CC, Carter BD, Feskanich D, Freedman ND, Prentice RL, Flanders WD: What proportion of cancer deaths in the contemporary United States is attributable to cigarette smoking? Ann Epidemio/ 2015, 25(3):179-182 e171.

4. Andreotti G, Freedman ND, Silverman DT, Lerro CC, Koutros S, Hartge P, Alavanja MC, Sandler DP, Freeman LB: Tobacco Use and Cancer Risk in the Agricultural Health Study. Cancer Epidemiol Biomarkers Prev 2017, 26(5):769-778. 
5. Levanen B, Glader P, Dahlen B, Billing B, Qvarfordt I, Palmberg L, Larsson K, Linden A: Impact of tobacco smoking on cytokine signaling via interleukin-17A in the peripheral airways. Int $J$ Chron Obstruct Pulmon Dis 2016, 11:2109-2116.

6. Menzies D, Nair A, Williamson PA, Schembri S, Al-Khairalla MZ, Barnes M, Fardon TC, McFarlane L, Magee GJ, Lipworth BJ: Respiratory symptoms, pulmonary function, and markers of inflammation among bar workers before and after a legislative ban on smoking in public places. JAMA 2006, 296(14):1742-1748.

7. Pleasance ED, Stephens PJ, O'Meara S, McBride DJ, Meynert A, Jones D, Lin ML, Beare D, Lau KW, Greenman $\mathrm{C}$ et al: A small-cell lung cancer genome with complex signatures of tobacco exposure. Nature 2010, 463(7278):184-190.

8. Wang XJ, Yang Y, Wang Z, Xu LL, Wu YB, Zhang J, Tong ZH, Shi HZ: Efficacy and safety of diagnostic thoracoscopy in undiagnosed pleural effusions. Respiration 2015, 90(3):251-255.

9. Li J, Wu H, Hao Y, Yao X: Unstable Carotid Plaque is Associated With Coagulation Function and Platelet Activity Evaluated by Thrombelastography. J Stroke Cerebrovasc Dis 2019:104336.

10. Luo H, Li X, Jiang A, Zhang B, Bi P, Dong Y, Guo Y: Associations of beta-Fibrinogen Polymorphisms with the Risk of Ischemic Stroke: A Meta-analysis. J Stroke Cerebrovasc Dis 2019, 28(2):243-250.

11. Cetin M, Erdogan T, Kiris T, Ozer S, Yilmaz AS, Durak H, Aykan AC, Satiroglu O: Predictive value of fibrinogen-to-albumin ratio in acute coronary syndrome. Herz 2019.

12. Chen Y, Zhu Y, Sheng Y, Xiao J, Xiao Y, Cheng N, Chai Y, Wu X, Zhang S, Xiang T: SIRT1 downregulated FGB expression to inhibit RCC tumorigenesis by destabilizing STAT3. Exp Cell Res 2019, 382(2):111466.

13. Li SQ, Jiang YH, Lin J, Zhang J, Sun F, Gao QF, Zhang L, Chen QG, Wang XZ, Ying HQ: Albumin-tofibrinogen ratio as a promising biomarker to predict clinical outcome of non-small cell lung cancer individuals. Cancer Med 2018, 7(4):1221-1231.

14. Cancer Genome Atlas Research N, Weinstein JN, Collisson EA, Mills GB, Shaw KR, Ozenberger BA, Ellrott K, Shmulevich I, Sander C, Stuart JM: The Cancer Genome Atlas Pan-Cancer analysis project. Nat Genet 2013, 45(10):1113-1120.

15. Gyorffy B, Surowiak P, Budczies J, Lanczky A: Online survival analysis software to assess the prognostic value of biomarkers using transcriptomic data in non-small-cell lung cancer. PLOS One 2013, 8(12):e82241.

16. Secretan B, Straif K, Baan R, Grosse Y, El Ghissassi F, Bouvard V, Benbrahim-Tallaa L, Guha N, Freeman $C$, Galichet $L$ et al: A review of human carcinogens-Part E: tobacco, areca nut, alcohol, coal smoke, and salted fish. Lancet Oncol 2009, 10(11):1033-1034.

17. Alexandrov LB, Ju YS, Haase K, Van Loo P, Martincorena I, Nik-Zainal S, Totoki Y, Fujimoto A, Nakagawa $\mathrm{H}$, Shibata $\mathrm{T}$ et al: Mutational signatures associated with tobacco smoking in human cancer. Science 2016, 354(6312):618-622.

18. Chapman AM, Sun KY, Ruestow P, Cowan DM, Madl AK: Lung cancer mutation profile of EGFR, ALK, and KRAS: Meta-analysis and comparison of never and ever smokers. Lung Cancer 2016, 102:122- 
134.

19. He J, Zeng ZC, Xiang ZL, Yang P: Mass spectrometry-based serum peptide profiling in hepatocellular carcinoma with bone metastasis. World J Gastroenterol 2014, 20(11):3025-3032.

20. Uccella S, Cromi A, Vigetti D, Cimetti L, Deleonibus S, Casarin J, Passi A, Riva C, Ghezzi F: Endometrial cancer cells can express fibrinogen: Immunohistochemistry and RT-PCR analysis. $J$ Obstet Gynaecol 2016, 36(3):353-358.

21. Alexander M, Wolfe R, Ball D, Manser R, McManus M, Riedel B, Solomon B, Westerman D, Burbury K: OC-05 - D-Dimer, fibrinogen and TEG-MA predict thromboembolism in non-small cell lung cancer interim results from a prospective cohort study. Thromb Res 2016, 140 Suppl 1:S170.

\section{Tables}

Table 1 Correlation between FGB expression levels and clinicopathological characters in LUAD 


\begin{tabular}{|c|c|c|c|c|}
\hline \multirow{3}{*}{$\begin{array}{c}\text { Clinical } \\
\text { Characters }\end{array}$} & \multicolumn{4}{|c|}{ Lung adenocarcinoma } \\
\hline & \multirow{2}{*}{$\begin{array}{l}\text { Cases } \\
\mathrm{N}=439\end{array}$} & \multicolumn{2}{|c|}{ FGB Expression } & \multirow[t]{2}{*}{$P$ value } \\
\hline & & Low & High & \\
\hline Age(years) ${ }^{\text {a. b. }}$ & & & & 0.792 \\
\hline$\leq 66$ & 200 & 118 & 82 & \\
\hline$>66$ & 220 & 127 & 93 & \\
\hline Smoking ${ }^{c}$. & & & & $<0.001 * * *$ \\
\hline Non-smoker & 179 & 124 & 55 & \\
\hline Smoker & 260 & 132 & 128 & \\
\hline T status & & & & 0.431 \\
\hline $\mathrm{pT}_{1}$ & 136 & 81 & 55 & \\
\hline $\mathrm{pT}_{2}$ & 245 & 137 & 108 & \\
\hline $\mathrm{pT}_{3-4}$ & 57 & 37 & 20 & \\
\hline N status & & & & $0.033 *$ \\
\hline $\mathrm{pN}_{0}$ & 289 & 179 & 110 & \\
\hline $\mathrm{pN}_{1-3}$ & 150 & 77 & 73 & \\
\hline M Status & & & & 0.876 \\
\hline $\mathrm{pM}_{0}$ & 419 & 244 & 175 & \\
\hline $\mathrm{pM}_{1}$ & 20 & 12 & 8 & \\
\hline
\end{tabular}




\begin{tabular}{ccccc} 
TNM Stage & & & & $0.046^{*}$ \\
I-II & 342 & 208 & 134 & \\
III-IV & 97 & 48 & 49 & \\
\hline
\end{tabular}

1. Median age was 66 years old.

2. Nineteen cases with unclear age features were no included.

3. Non-smoker included those never smokers and current reformed smoker > 15years; Smokers include those current reformed smoker $\leq 15 y e a r s$ and current smoker.

$P$ value was evaluated by Chi-square test. *, $P<0.05 ; * *, P<0.01 ; * *, P<0.001$.

Table 2 Multivariable Cox-regression analysis for OS and PFS in LUAD

\begin{tabular}{lccccccc}
\hline \multirow{2}{*}{ Factors } & \multicolumn{4}{c}{ OS } & & \multicolumn{3}{c}{ PFS } \\
\cline { 2 - 4 } \cline { 6 - 8 } & HR & $(95 \%)$ CI & P value & & HR & $(95 \%)$ CI & P value \\
\hline Age & 1.208 & $0.801-1.823$ & 0.367 & & 1.607 & $0.953-2.709$ & 0.075 \\
Smoking & 0.902 & $0.734-1.109$ & 0.329 & & 0.993 & $0.764-1.290$ & 0.958 \\
\hline Stage & 1.646 & $1.368-1.981$ & 0.000 & & 1.239 & $0.969-1.585$ & 0.088 \\
& & & & & & \\
FGB & 1.616 & $1.090-2.395$ & 0.017 & & 1.710 & $1.060-2.759$ & 0.028 \\
\hline
\end{tabular}

$H R$ hazard ratio, $C I$ confidence interval

Table 3 Multivariable Cox-regression analysis for OS and PFS in LUAD with tobacco exposure

\begin{tabular}{lccccccc}
\hline Factors & \multicolumn{4}{c}{ OS } & & \multicolumn{3}{c}{ PFS } \\
\cline { 2 - 3 } \cline { 6 - 8 } & HR & $(95 \%)$ CI & P value & & HR & (95\%)CI & P value \\
\hline Age & 1.416 & $0.865-2.321$ & 0.167 & & 2.351 & $1.277-4.328$ & 0.006 \\
\hline Stage & 1.529 & $1.231-1.898$ & 0.000 & & 1.388 & $1.046-1.843$ & 0.023 \\
& & & & & & & \\
FGB & 1.806 & $1.071-3.044$ & 0.027 & & 2.181 & $1.182-4.023$ & 0.013 \\
\hline
\end{tabular}

$H R$ hazard ratio, $C I$ confidence interval

\section{Figures}


A

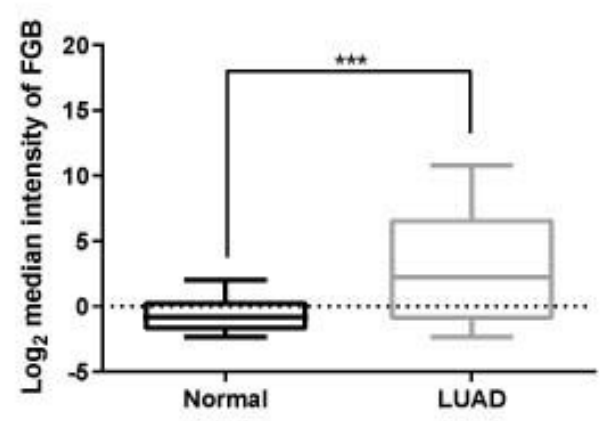

B

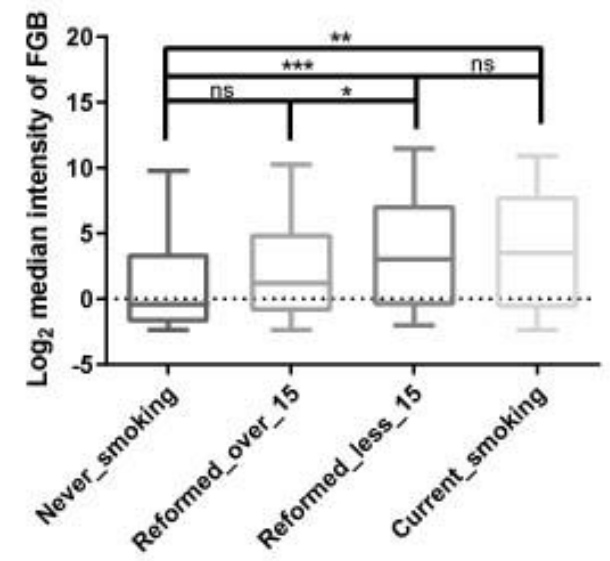

Figure 1

Expression pattern of FGB in LUAD. (A) FGB was significantly overexpressed in tissues compared with in normal tissues. (B) The expression level of FGB in those reformed smoker less than fifteen years and current smoker was higher than in those reformed smoker more than fifteen years and never smoker.

A

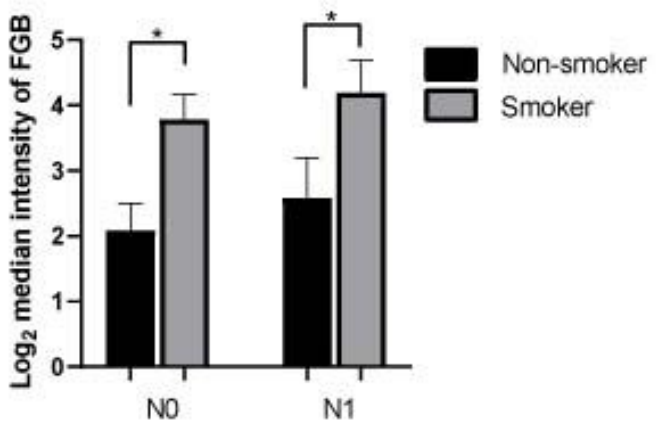

B

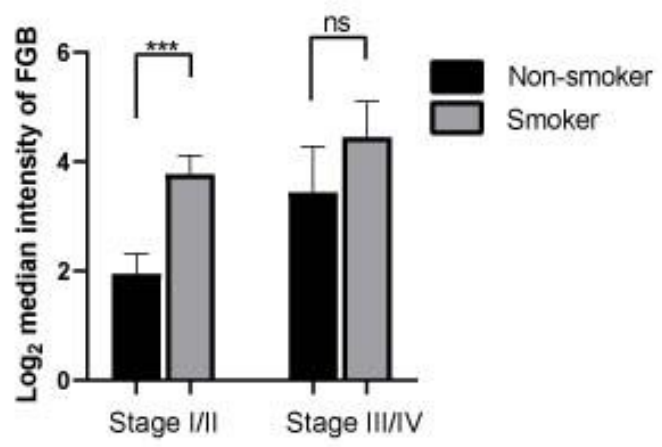

Figure 2 
Correlation between tobacco exposure and clinical features. (A) The smoker group possessed higher level of FGB both in LUAD patients without $(P=0.003)$ or with $(P=0.045)$ lymph node metastasis. (B) The smoker group possessed higher level of FGB in stage I/II LUAD patients $(P=0.0004)$, but not in stage III/IV patients $(P=0.350)$.

A

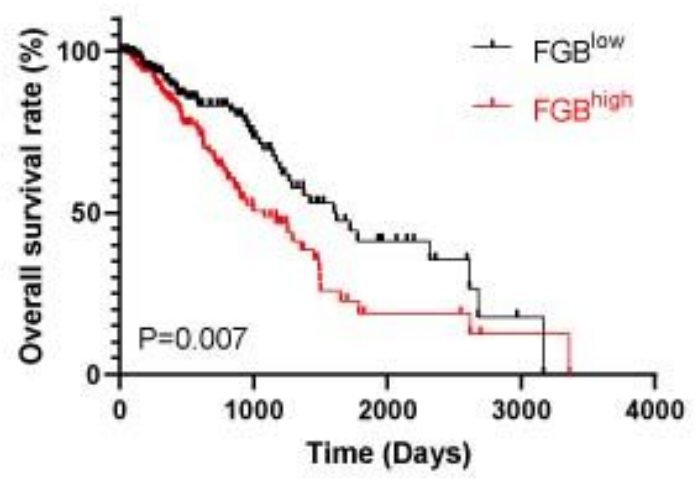

B

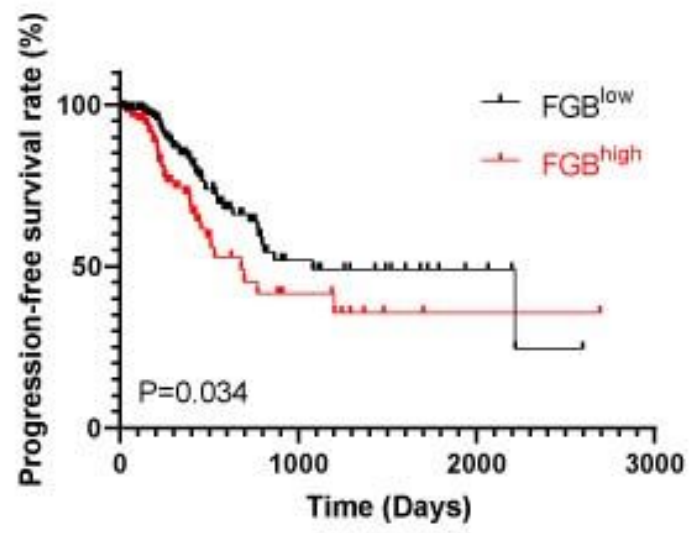

Figure 3

Prognostic value of FGB in LUAD patients. Kaplan-Meier survival curves generated from TCGA cohort indicated that elevated expression of FGB predicted worse overall survival (A) and progression-free survival (B). 
A

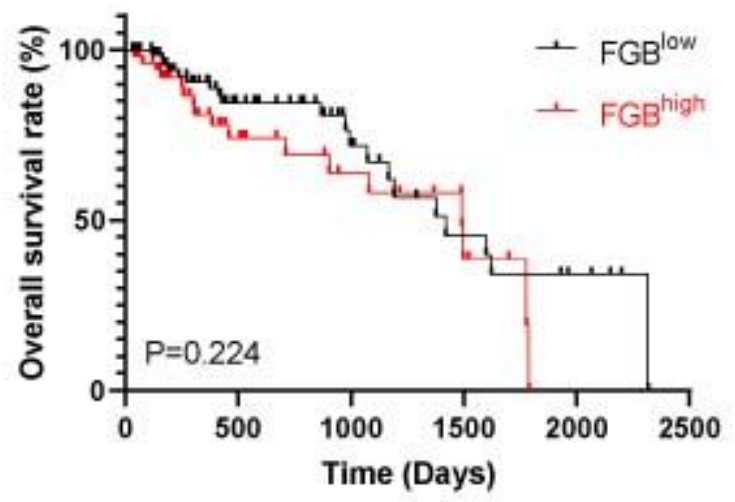

$\mathrm{C}$

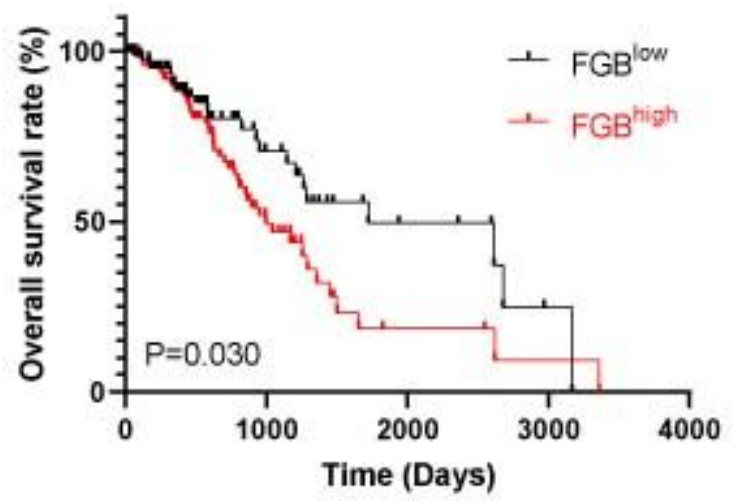

$\mathrm{B}$

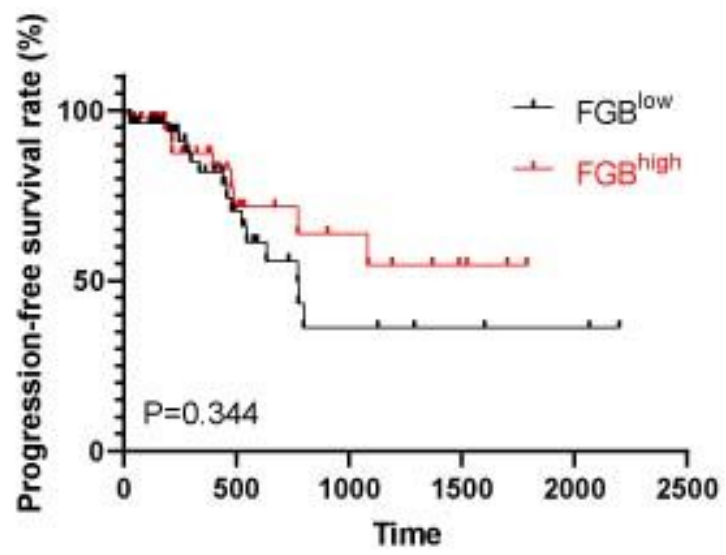

$\mathrm{D}$

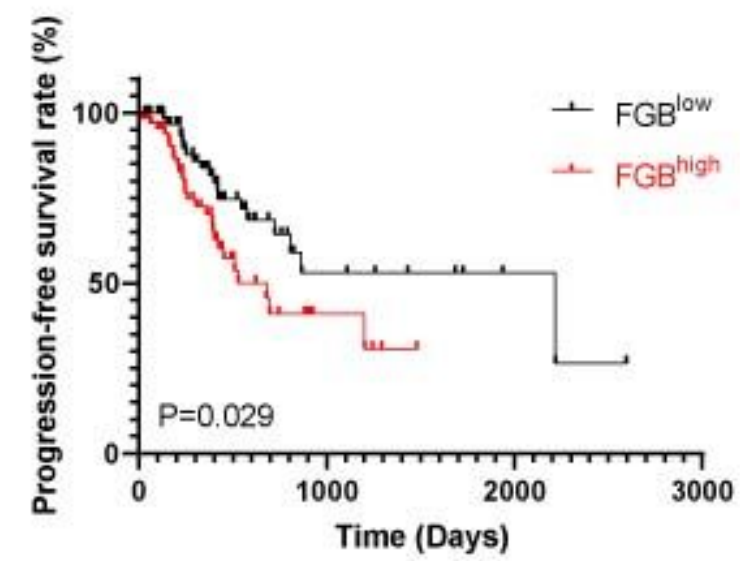

\section{Figure 4}

Prognostic value of FGB in LUAD patients stratified by smoking status. No significant association between FGB expression and patient OS (A) and PFS (B) were observed in non-smoker group. However, high level of FGB expression predicted worse OS (C) and PFS (D) in the smoker group. 
A

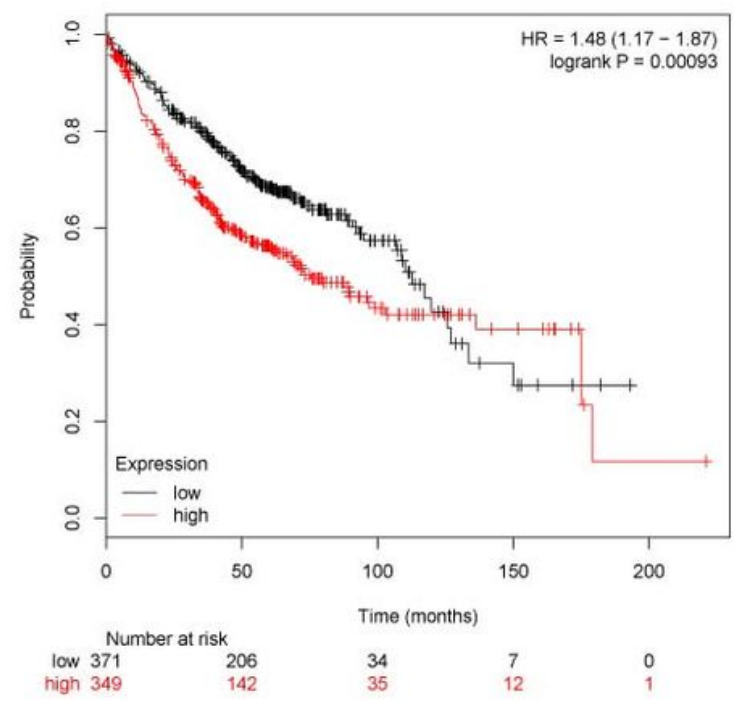

B 216238_s_at

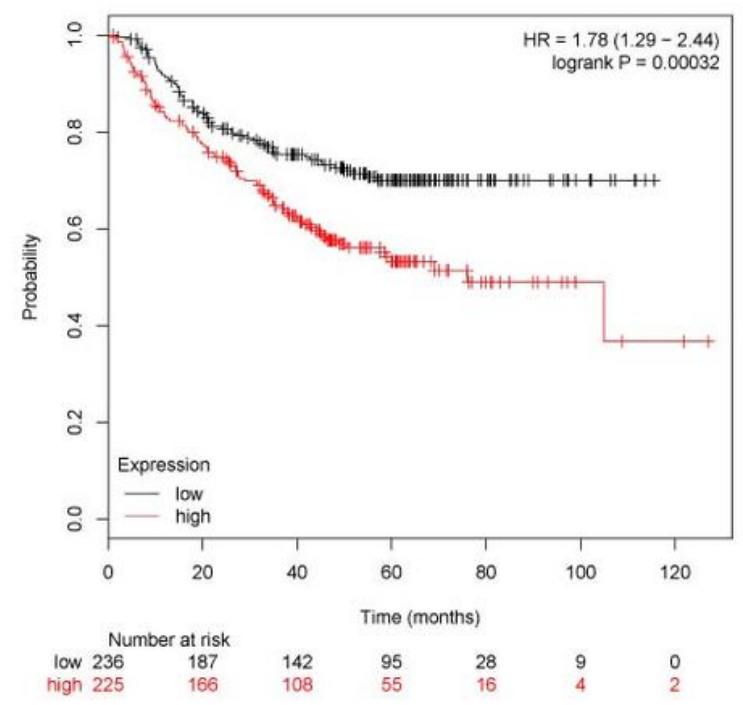

\section{Figure 5}

The prognostic effect of FGB in LUAD patients in KM plotter cohort. The desired Affymetrix IDs is valid: 216238_S_at (FGB). Elevated expression of FGB predicts worse prognosis of overall survival (AØand progression-free survival (B). 
A

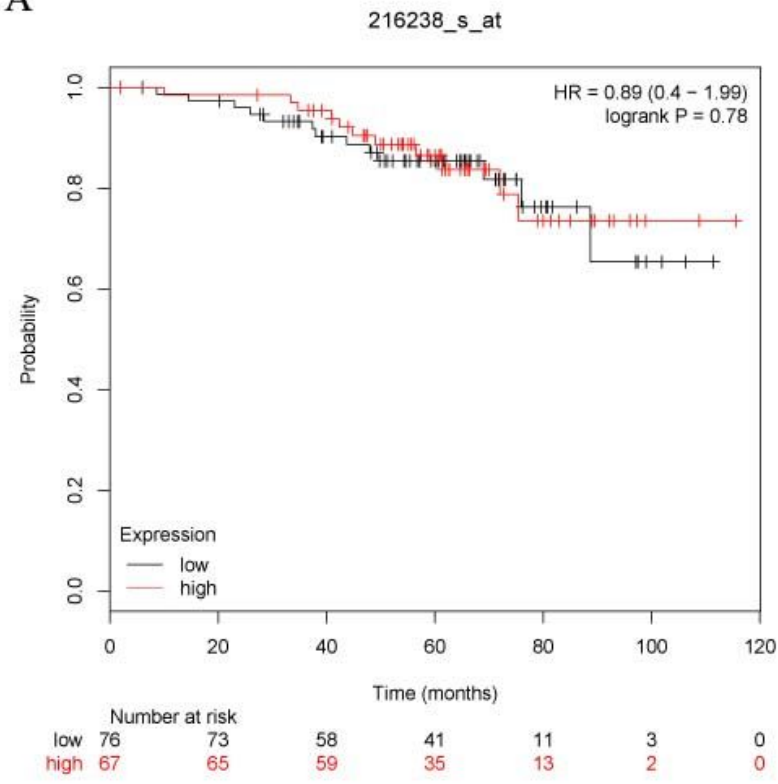

C

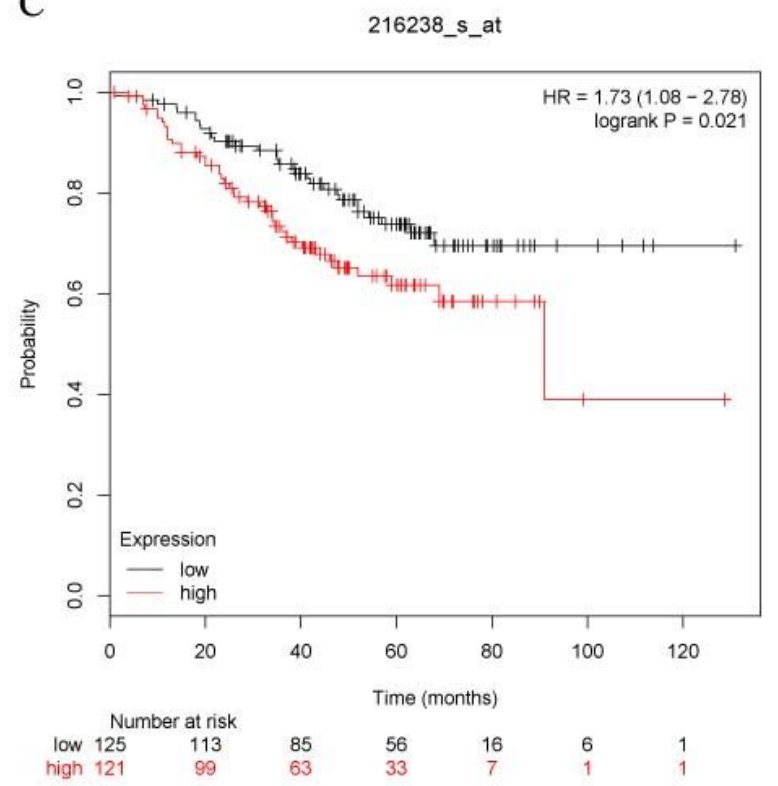

B

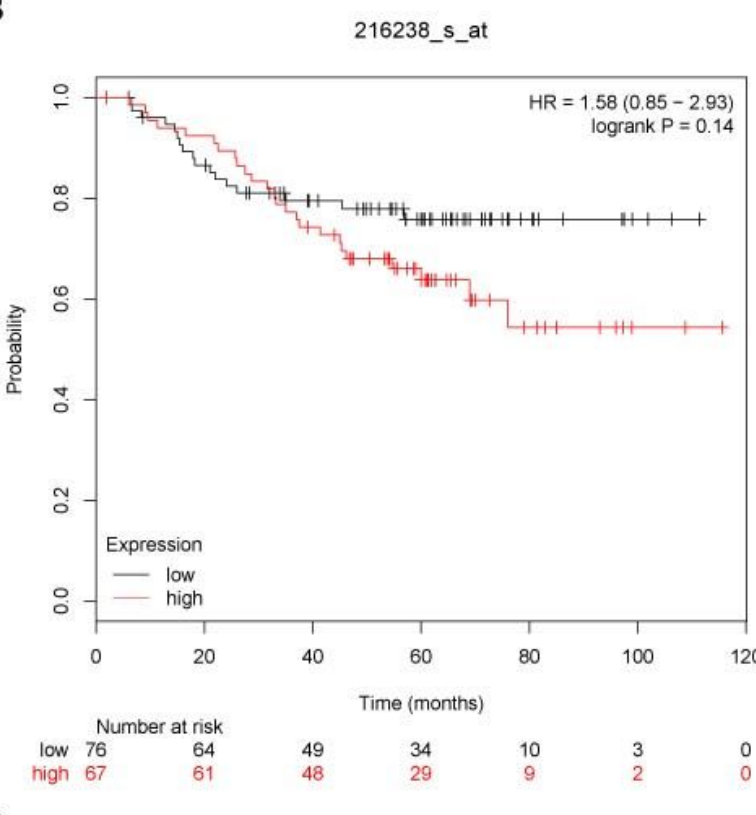

D

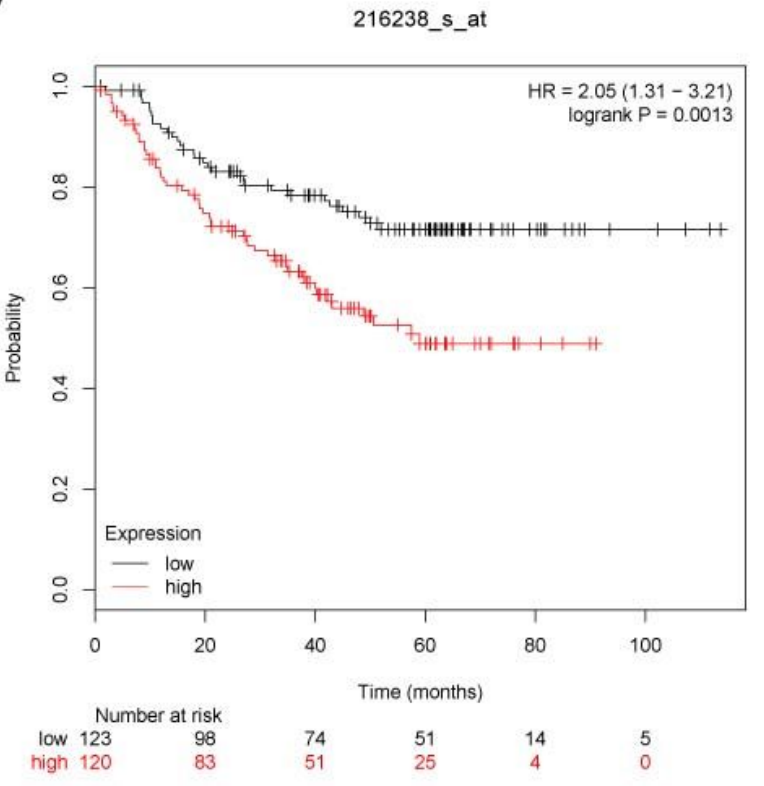

\section{Figure 6}

The prognostic effect of FGB in LUAD patients in KM plotter cohort stratified by smoking status. The desired Affymetrix IDs is valid: 216238_s_at (FGB). No significant association between FGB expression and patient OS (A) and PFS (B) were observed in LUAD patients without tobacco exposure. However, in LUAD patients with tobacco exposure, high level of FGB expression predicted worse OS (C) and PFS (D). 